Textures and Microstructures, 1991, Vols 14-18, pp. 193-197 Reprints available from the publisher

Photocopying permitted by license only (c) 1991 Gordon and Breach Science Publishers SA Printed in the United Kingdom

\title{
EVALUATION OF THE PARAMETERS OF TEXTURE COMPONENTS ON THE BASIS OF A DISCRETE FORM OF ORIENTATION DISTRIBUTION
}

\author{
J . JURA
}

Aleksander Krupkowski Institute for Metal Research Polish Academy of Sciences

ul. Reymonta 25, 30-059 Krakow, Poland

\section{INTRODUCTION}

A method is proposed which enables to evaluate the parameters of the texture components (i.e. volume fraction and the scattering width around the ideal orientation) on the basis of orientation distribution given in a discrete form. The presented method is a continuation and generalisation of the evaluation method applied so far ${ }^{2}$, in which the coefficients of a series expansion of the orientation distribution function (ODF) are used. The old method may be now utilized as a preliminary stage in the determination of of the parameter values.

\section{TEXTURE COMPONENT}

As a texture component we mean the orientation $g_{i}=$ (hkl)[uvw] together with its surroudings, in which there occurs increased orientation density. A simple description of a texture component in the position $g_{i}$ is obtained by assuming that all the orientations $g$ from the surroudings $g$ defined by the rotation of a crystallite with the orientation $g$, around an arbitrary rotation axis $\bar{a}$ by the same rotation angle $\omega_{c}$ are equally probable

$$
f_{i}(g)=f\left(\left\{\bar{a}, \omega_{c}\right\}\right)=\text { const. } ; \quad \omega=\left|g_{i}^{-1} \times g\right| .
$$

If there exists a symmetry of the crystallographic lattice and a statistical symmetry of the sample of the examined material, all the symmetrically equivalent orientations should be considered.

The $f_{i}(g)$ function defined that way is dependent only on the rotation angle $\omega$

$$
f_{i}(g)=S_{i}(\omega) \text {. }
$$


The normalisation coefficient of the function $S_{1}(\omega)$, called the scattering function, corresponds by definition to the volume fraction $w_{1}$ of the $i-t h$ component in the texture

$$
\oint f_{i}(g) d g=V_{i} / V=W_{i} .
$$

where $V$ - sample volume, $V_{1}$ - volume of crystallites attributed to the $i$ - th component.

The function $S_{i}(\omega)$ can be described by means of Gauss distribution

$$
S_{i}(\omega)=S_{0 i} \exp \left(-\omega^{2} / \omega_{0 i}^{2}\right)
$$

( after H.J.Bunge ${ }^{3}$ ) or

$s_{i}^{\prime}(\omega)=s_{0 i}^{\prime} \exp \left[s_{0 i} \cos (\omega)\right] ; s_{0 i}=\ln 2 /\left[2 \sin ^{2}\left(b_{0 i} / 4\right)\right]$

(after S.Matthies ${ }^{4}$ ).

In the relationships (3-5) $W_{i}$ denotes the volume fraction of the component $i,{ }^{i}$ and $\omega_{0}$ (or $b_{0}$ ) characterizes the scattering of the orientations around the position $g_{i}$. The parameters $W$ and $\omega$ ( $\omega_{0}$ ( ${ }^{\prime}$ ) component i.

MODEL ODF

In a general case, in the texture, there may occur several preferred orientations $\left\{g_{i} ; i=1, I\right\}$ surrounded by the scattering areas of high orientation densities. By adopting a mathematical description for the orientation distribution in each of these areas a model representation of the ODF $f_{\bmod }(g)$ is obtained

$$
f_{\text {mod }}(g)=\sum_{i=1}^{I} f_{i}(g)+r ; \quad r+\sum_{i=1}^{I} W_{i}=1,
$$

where $r$ represents the fraction of randomly distributed crystallites. The values of the parameters $\left\{w_{1}, \omega_{01} ; i=\right.$ $1, I\}$ and $r$ can be determined by a mean square approximation of the model distribution $f_{m o d}(g)$ to the distribution obtained on the basis of experimental data $f_{\text {exp }}(g)$

$$
\oint\left[f_{\text {exp }}(g)-f_{\text {mod }}(g)\right]^{2} d g=\min .
$$

Approximation of the function $f(g)$ by means of the function $f$ ( $g$ ) can be performed by using the expansion ${ }^{m} f^{d}$ each of the functions into a series of generalized spherical functions. Estimation of the 
value of $W_{1}$ and $\omega_{0 i}$ becomes then reduced to the approximation of the copfficients of the series expansion of $f_{\text {exp }}(g): C_{1 \text { exp }}$ by means of the coefficients of the function $f_{\text {mod }}(g)$ :
$C_{1 \bmod }^{\mu \nu}\left(r,\left\{W_{1}, \omega_{01}, g_{1} ; i=1, I\right\}\right) 1,2,5,6$.

Besides the methods of the ODF determination based on its series expansion, the so-called discrete methods of the ODF determination from pole figures are more and more frequently employed at present. These methods, supply a set of the $f_{\text {e }}\left(g_{j}\right)$ values at the points $g_{j}$ in the orientation space $(j=1, J)$. Evaluation of the parameters of the texture components may be then performed directly on the basis of relation (7) in which the integration is replaced by summing with the weight factors $w\left(g_{j}\right)$

$$
\sum_{j=1}^{J}\left[f_{e x p}\left(g_{j}\right)-f_{\text {mod }}\left(g_{j}\right)\right]^{2} w\left(g_{j}\right)=\text { min. }
$$

Such procedure allows to avoid the truncation error connected with breaking the ODF series expansion at $1_{\text {max }}$, where $1_{\text {ma }} \ll \infty$ ( 1 - series expansion order $)$. Also, mostly when using the ODF series expansion we have at our disposal only the coefficients $C_{1}$ exp for even value of $1\left(1=21^{\prime}\right)$, thus incomplete information about ODF. The discrete methods, on the other hand, provide more reliable data about the orientation disdistribution by eliminating the efffect of disturbances, the so-called "ghost phenomena"

\section{RESULTS}

As an example of the application of discrete data the relation ( 8 ) has been used to determine the parameters of texture components in a silver sample cold-rolled to $98.4 \%$ reduction . The ODF has been reproduced by the $A D C$ method . The results of fitting have been compared with the values obtained by the method utilizing the coefficients of the ODF series expansion (Table 1). It is observed that for the main texture components both methods yield similar values of the parameters, but considerable differences occur for the minor components and the fraction of randomly distributed crystallites. The differences are due to high relative errors of the low orientation densities when the series expansion method is used. Figure 1a shows sections of the ODFs obtained by discrete method, while figure $1 \mathrm{~b}$-those calculated and fitted by the series expansion method. 
a)

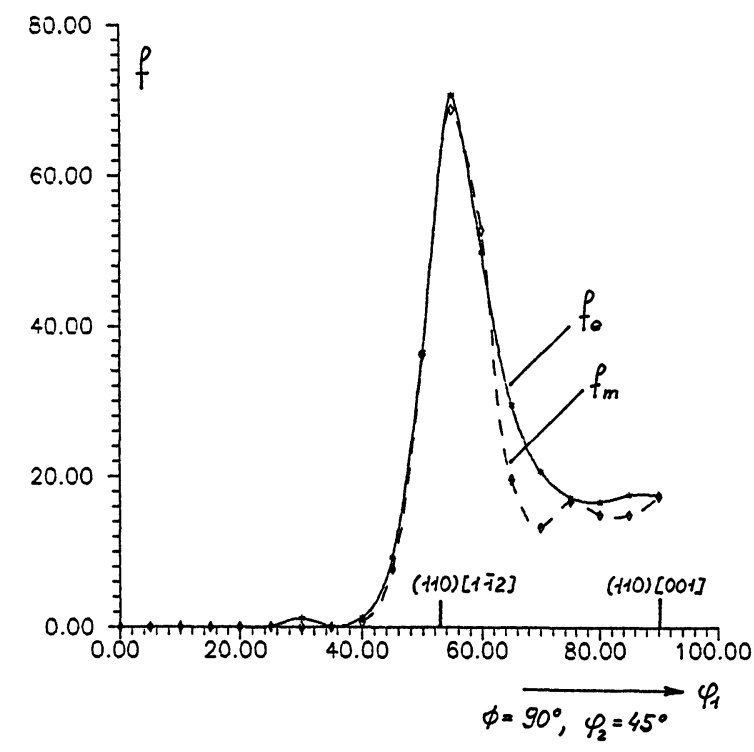

b)

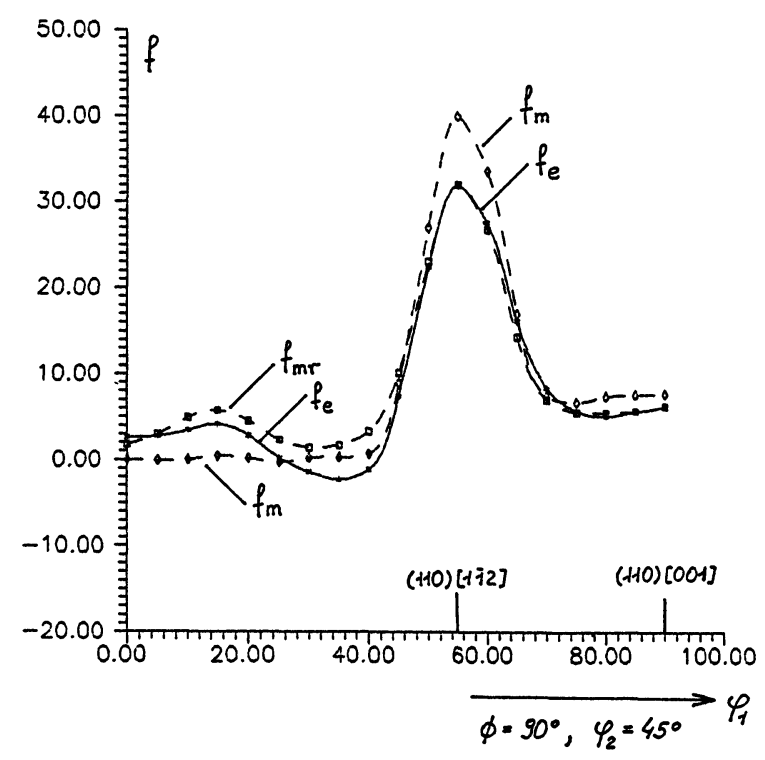

Fig. 1 Sections through the ODF of a $98.4 \%$ cold-rolled silver.

a) The discrete method has been applied: $f$-experimental ODF, $f$ - model ODF calculated with component paramete ${ }^{m}$ s from Table 1 .

b) The series expansion method has been used: $f$-experimental ODF $\left(1_{1}=22\right)$ including only 1 -even têrms, f -model oDF including only l-even terms, $f$-model ${ }^{m r}$ ODF including 1 -even and 1 -odd terms (' component parameters from Table 1.). 
Table 1. Comparison of the values of the texture component parameters

$$
\begin{aligned}
& \text { Discrete method Series } \\
& \text { expansion } \\
& \text { method }
\end{aligned}
$$

\begin{tabular}{crrrrrrr} 
Component & $\varphi_{1}$ & ${ }_{0}$ & \multicolumn{1}{c}{$\varphi_{2}$} & $W[\%]$ & $\omega_{0}\left[{ }^{0}\right]$ & $W[\%]$ & $\omega_{0}\left[{ }^{0}\right]$ \\
$r$ & - & - & - & 0.0 & - & 16.4 & - \\
$\{110\}<112\rangle$ & 35.0 & 45.0 & 0.0 & 50.4 & 7.4 & 47.2 & 8.4 \\
$\{110\}<001\rangle$ & 0.0 & 45.0 & 0.0 & 8.0 & 8.2 & 6.0 & 9.6 \\
$\sim\{110\}<115\rangle$ & 15.0 & 45.0 & 0.0 & 17.3 & 8.4 & 7.0 & 8.3 \\
$\sim\{627\}<496\rangle$ & 50.0 & 40.0 & 70.0 & 24.2 & 8.8 & 23.5 & 8.1
\end{tabular}

\section{SUMMARY}

The new method has been applied to determine the parameters of texture components. It allows to characterize the texture by a few numbers only which define the position of the main components and the values of their parameters (volume fractions and scattering widths, as well as the fraction of randomly distributed crystallites. The discrete representation of the ODF provides more reliable data for approximation and the parameter values are then also fitted more precisely.

\section{REFERENCES}

1 W.Truszkowski, J.Pospiech, J.Jura and B.Major, Proc. $3^{\mathrm{e}}$ colloque europeen sur les textures de deformation et de recristallisation des metaux et leurs applications industrielles Pont-a-Mousson 235 (1973)

2 J.Pospiech, K.Lücke and J.Jura, Proc. 6th International Conference on Textures of Materials Tokio (1981) 1390

3 H.J.Bunge, Texture Analysis in Materials Sciences Butterworths, London 1982

4 S.Matthies, G.W.Vinel and K.Helming, Standard Distributions in Texture Analysis Akademie-Verlag, Berlin 1987

5 J.Jura, K.Lücke and U.v.Schlippenbach, Proc. 8th International Conference on Textures of Materials Santa Fe 123 (1987)

6 J.Jura, Bull.Pol Ac.: Tech. 36,183 (1988)

7 S.Matthies, G.W.Vinel, phys.stat.sol.(b) 112, K111K114

8 K.Pawlik, phys.stat.sol.(b) 134,477 (1986)

9 J.Jura, Proc. XIVth Conference on Applied Crystallography Cieszyn-Poland 66 (1990) 